\title{
Fabrication of Amorphous Micro-Ring Arrays in Crystalline Silicon Using Ultrashort Laser Pulses
}

\author{
Yasser Fuentes-Edfuf ${ }^{1,}$, , Mario Garcia-Lechuga ${ }^{1}$, Daniel Puerto ${ }^{1}$, Camilo Florian ${ }^{1}$, Adianez \\ Garcia-Leis ${ }^{2}$, Santiago Sanchez-Cortes ${ }^{2}$, Javier Solis ${ }^{1}$, and Jan Siegel ${ }^{1, *}$
}

1. Laser Processing Group, Instituto de Óptica, CSIC, Serrano 121, 28006 Madrid, Spain

2. Instituto de Estructura de la Materia, CSIC, Serrano 121, 28006 Madrid, Spain

*corresponding authors: y.fuentes@io.cfmac.csic.es, j.siegel@csic.es

Keywords: Semiconductors; Phase transformation; Femtosecond laser processing; Nanopatterning; Pump-probe microscopy; Silicon; Micro-Raman spectroscopy;

\begin{abstract}
:
We demonstrate a simple way to fabricate amorphous micro-rings in crystalline silicon using direct laser writing. The method is based on the fact that the phase of a thin surface layer can be changed into the amorphous phase by irradiation with a few ultrashort laser pulses $(800 \mathrm{~nm}$ wavelength and $100 \mathrm{fs}$ duration). Surface-depressed amorphous rings with a central crystalline disk can be fabricated without the need for beam shaping, featuring attractive optical, topographical, and electrical properties. The underlying formation mechanism and phase change path way have been investigated by means of fs-resolved microscopy, identifying fluence-dependent melting and solidification dynamics of the material as the responsible mechanism. We demonstrate that the lateral dimensions of the rings can be scaled and that the rings can be stitched together, forming extended arrays of structures not limited to annular shapes. This technique and the resulting structures may find applications in a variety of fields in optics, nanoelectronics, and mechatronics.
\end{abstract}

\section{Text:}

Silicon is more than just a key material for the electronics industry; it can be considered one of the pillars the industry is built on. Si owes this position essentially to its abundance on earth, its semiconducting properties and the existence of two structurally different solid phases (crystalline and amorphous), having very different physical properties. In this context it is worth noting that the amorphous phase can be formed by a variety of methods, including chemical and physical vapour deposition techniques, ion implantation and melting followed by rapid quenching. While some of the properties of the amorphous phase are known to depend on the preparation method ${ }^{1}$ it has to be kept in mind that it is still a tetrahedrally coordinated semiconductor with a coordination number close to four ${ }^{2}$, just as the crystalline phase.

Laser processing of Si started immediately after sufficiently intense lasers were available ${ }^{3}$ and today applications are countless. The ability of pulsed laser irradiation to melt the crystalline material and induce resolidification either into the crystalline or amorphous phase, depending on the irradiation conditions, has been observed already in 1979 by Liu et al ${ }^{4}$. In fact, the authors proposed this concept for data storage applications due to the optical contrast between the two phases. Several experimental parameters influence the melting and freezing kinetics of the material and thus the final phase obtained, the most important ones being the

Appl. Phys. Lett. 110, 211602 (2017); doi: http://dx.doi.org/10.1063/1.4984110 
laser pulse duration, wavelength and number of irradiation pulses, as well as film thickness and choice of substrate in the case of thin Si films ${ }^{5-10}$.

Despite this early discovery, most laser-induced phase-change studies in Si focused on transforming large areas, for instance laser-annealing of amorphous Si for fabrication of solar cells $s^{11, \underline{12}}$ or OLED displays ${ }^{\underline{13}}$. Less work has been done to imprint phase-change micro- or nanostructures into an homogeneous Si background. Laser interference patterning of a-Si to form parallel crystalline lines and dots with sub-wavelength separation was reported in $\underline{14}$. Recently, direct writing of crystalline lines in hydrogenated amorphous Si films was reported, which can be considered as semiconductive channels in an insulating matrix, due to the enormous difference in electrical conductivity of both phases $\frac{15}{}$. The inverse process, direct laser writing of amorphous structures in crystalline $\mathrm{Si}$ by high repetition rate femtosecond laser irradiation was reported by Kiani et al $\underline{16}, \underline{17}$. Using nanosecond laser irradiation, Pena et al. produced micrometer-sized amorphous humps with a height of a few nanometers $\frac{18}{}$. Recently, it has been shown that Laser-Induced Periodic Surface Structures (LIPSS) in form of alternating amorphous and crystalline fringes can be fabricated by scanning the laser beam over the sample surface, employing an adequate choice of spot size, repetition rate and scan velocity $\underline{19,20}$. In the present work we present a simple strategy to fabricate surface-depressed annular amorphous rings with a central crystalline disk, temporally resolve their formation process, and show how these structures can be scaled and stitched together to form arrays with different symmetries.

The sample used was a commercial $<100>$ oriented crystalline silicon wafer with $p$-doping (boron, resistivity 1-5 $\Omega \mathrm{cm}$ ). Single or multiple fs laser pulses (120 fs duration at $800 \mathrm{~nm}$ wavelength, p-polarized) were sent through a circular aperture with a diameter $\varnothing=3.5 \mathrm{~mm}$ before being focused by a lens with focal length $\mathrm{f}=150 \mathrm{~mm}$ at the sample surface, at an angle of incidence $\theta$. The intensity distribution at the sample plane was measured to be Gaussian with a spot diameter of $d=59 \mu \mathrm{m}\left(1 / \mathrm{e}^{2} \text {-diameter }\right)^{21}$. Figure 1 shows optical microscopy images $(100 \mathrm{x}$ objective, $\mathrm{NA}=0.9)$ of the $\mathrm{Si}$ sample after exposure to a different number of laser pulses at a constant fluence $F=250 \mathrm{~mJ} / \mathrm{cm}^{2}$. As the pulse number $\mathrm{N}$ increases, an annular structure emerges, featuring a ring of increased reflectivity and a central disk of similar reflectivity as the non-exposed region (Fig. 1(a-c)). The reflectivity increase within the annular region is consistent with surface amorphization $\underline{22}, \underline{23}$, since the absorption coefficient of the amorphous phase of $\mathrm{Si}$ at the illumination wavelength is known to be much higher than that of the crystalline phase, leading to a reflectivity increase. The formation of a silicon oxide layer thick enough to affect the optical properties of the irradiated region has been discarded by EDX measurements indicating an oxygen content similar to that of the non-irradiated material.

As demonstrated for single pulse irradiation of $\mathrm{Si}<111>\underline{22,23}$, the thickness of the amorphous layer formed can be estimated by modeling a multilayer system based on Abèles theory ${ }^{24}$ using the optical constants of both phases ${ }^{25}$. The result of the calculation is shown in Fig. 1(e), representing the calculated reflectivity change as a function of thickness of the amorphous top layer. The oscillatory behavior of the curve is caused by interference of the light reflected at the surface with light reflected at the amorphous-crystalline interface. The damping behavior is due to the elevated $k$-value of the amorphous phase.

In order to estimate the maximum thickness of the amorphous layer for each irradiation we have extracted the corresponding radial reflectivity cross sections from the images and determined the reflectivity change value in the center of the annulus (marked by star symbols in Fig. 1(a-d)). In a second step, we have identified these values in the calculated curve in Fig. 1(e), featuring the corresponding star symbols. Two immediate conclusions can be drawn. First, an excellent agreement of the maximum reflectivity change observed experimentally for $\mathrm{N}=5$ with the calculation is found, confirming that the annular structures consist of an 
amorphous surface layer. Second, the thickness of the amorphous top layer is shallow and an increasing function of pulse number $\mathrm{N}$. This accumulative behavior is quite similar to the well-known effect of a reduction of the ablation threshold for increasing pulse numbers, both being caused by an increase of the absorption coefficient and therefore an enhanced energy deposition during the first pulses. The thickness of laser-induced amorphous layers in Si has been investigated by Izawa et al. using transmission electron microscopy performed at sample cross sections ${ }^{\underline{8}}$. Using the same irradiation wavelength and pulse duration as ours, the authors reported a maximum thickness of $40 \mathrm{~nm}$ for $\mathrm{N}=20$ (the only $\mathrm{N}$ value they studied). It has to be kept in mind, though, that the authors did not discuss the lateral (local fluence) dependence of the amorphous layer thickness. In contrast, our optical microscopy technique probes the thickness at each position and provides a reflectivity value, whose thickness correspondence can be determined via the curve in Fig. 1(e).
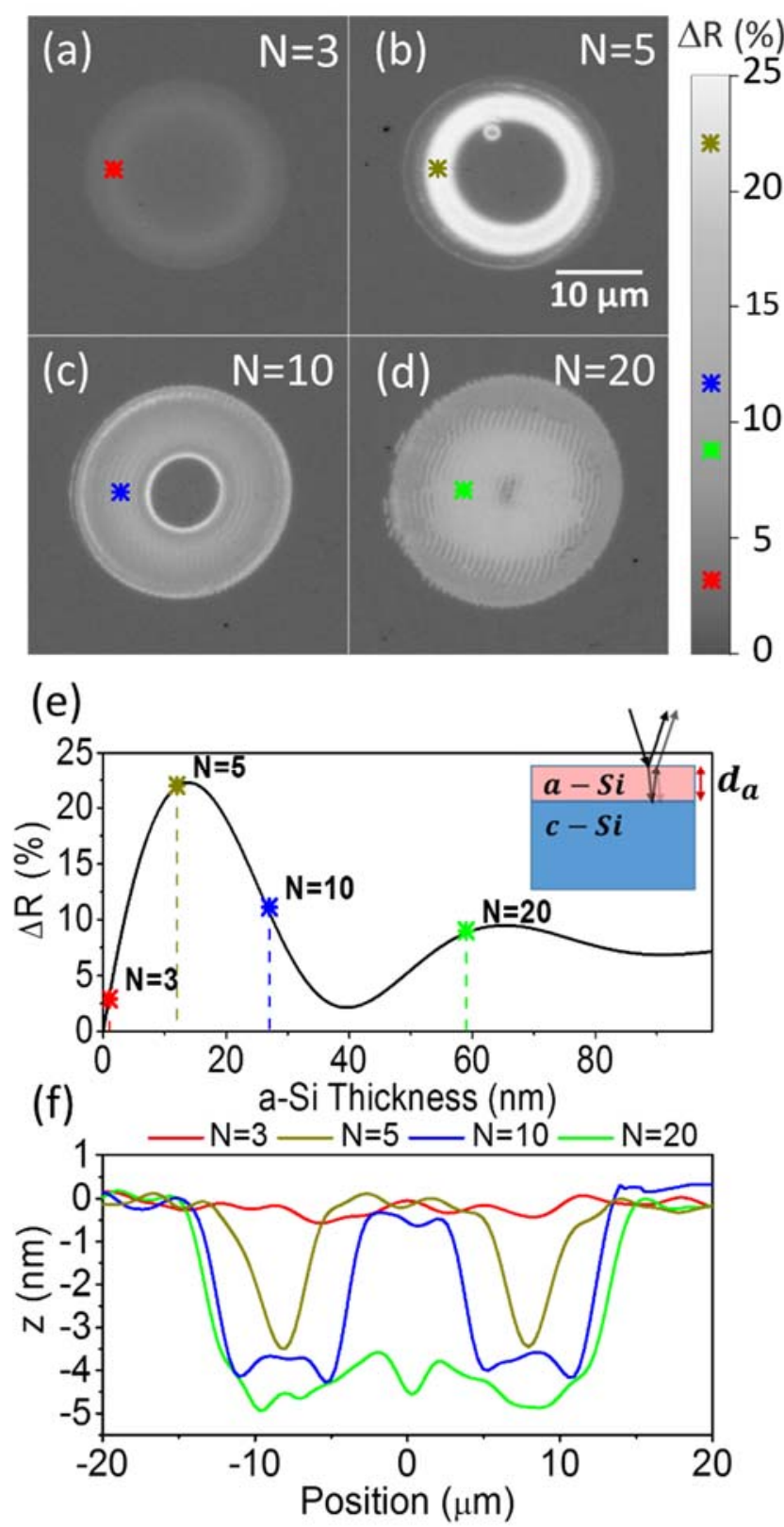

Figure 1: Optical micrographs of annular structures fabricated in Si by irradiation with (a) $\mathrm{N}=3$, (b) $\mathrm{N}=5$, (c) $\mathrm{N}=10$ and (d) $\mathrm{N}=20$ laser pulses at normal incidence $\left(\theta=0^{\circ}\right)$, using 
a Gaussian intensity distribution with a spot size of $d_{x}=d_{y}=59 \mu \mathrm{m}$ and a peak fluence $\mathrm{F}=$ $250 \mathrm{~mJ} / \mathrm{cm}^{2}$. The grey scale indicate the reflectivity change $\Delta \mathrm{R}$ induced by the laser. The star symbols mark the position for which the determined reflectivity change values have been plotted in (e). (e) Calculated reflectivity change as a function of thickness of an amorphous top layer, as indicated in the inset and using the model described in the text, including the experimentally determined reflectivity change values extracted from (a-d, star symbols). (f) Cross sections of the surface topography through the center of annular structures (a-d) fabricated by different numbers of laser pulses (see legend).

We interpret the appearance of the amorphous phase as being caused by laser induced melting followed by rapid solidification. While the above results demonstrate the amorphous nature of the annular structure, the nature of the central disk in Fig. 1(a-c) is not yet clear. The absence of a permanent reflectivity change suggests the central disk region to be crystalline. Yet, such interpretation might seem in conflict with the fact that the central region has been exposed to a higher local fluence than the annular region. In the following, we show that this intriguing phenomenon can be understood by considering the fluence-dependent solidification dynamics of the laser-molten material.

Pump-probe experiments have been performed in order to verify the occurrence of melting also in the central disk region. Exploiting the fact that the transient liquid phase of Si is metallike and thus has a high reflectivity ${ }^{26-28}$, conclusions about the presence and duration of the melting process can be drawn by measuring the transient reflectivity of the sample immediately after the arrival of the pump pulse. To this end, a convenient experimental technique is fs microscopy, which consists of a fs probe pulse illuminating the sample at a given optical delay and the recording of the transient reflectivity image with a conventional CCD camera $\underline{24,27,28}$. In order to perform this measurement, laser irradiation was incident at $\theta=$ $52^{\circ}$, yielding an elliptic spot size $\left(d_{x}=103 \mu \mathrm{m}\right.$ and $\left.d_{y}=59 \mu \mathrm{m}\right)$. Illumination was performed with a $400 \mathrm{~nm}$ fs probe pulse, produced by frequency-doubling a fraction of the pump pulse. The exposed region of the sample is monitored in-situ with an optical microscope, consisting of a microscope objective $(\mathrm{MO}, 20 \times$, N.A. $=0.42)$, a tube lens and a CCD camera, protected by a filter to block the pump light. The delay between pump and probe was controlled by means of a motorized optical delay line.

A consequence of performing irradiation at oblique incidence was that fewer pulses $(\mathrm{N}=3)$ were needed to generate an amorphous ring than for normal incidence $(\mathrm{N}=5)$, most likely due to the different local energy coupling for polarized light at oblique incidence. Fig. 2 shows selected time-resolved images at different stages of the melting and solidification process. At $\mathrm{t}=100$ ps (Fig. 2(a)), a large elliptical region of high transient reflectivity is observed, having an almost identical lateral extension as the elliptical ring of enhanced permanent reflectivity that appears in the final image recorded at long delays (Fig. 2(c), $t=$ inf.). This strong reflectivity increase confirms the presence of the liquid phase and its measured amplitude $(\Delta R \approx 0.45$, c.f. Fig. $2(\mathrm{~d}))$ matches well the value obtained by calculation, using the optical properties of liquid $\mathrm{Si}^{26}$. 


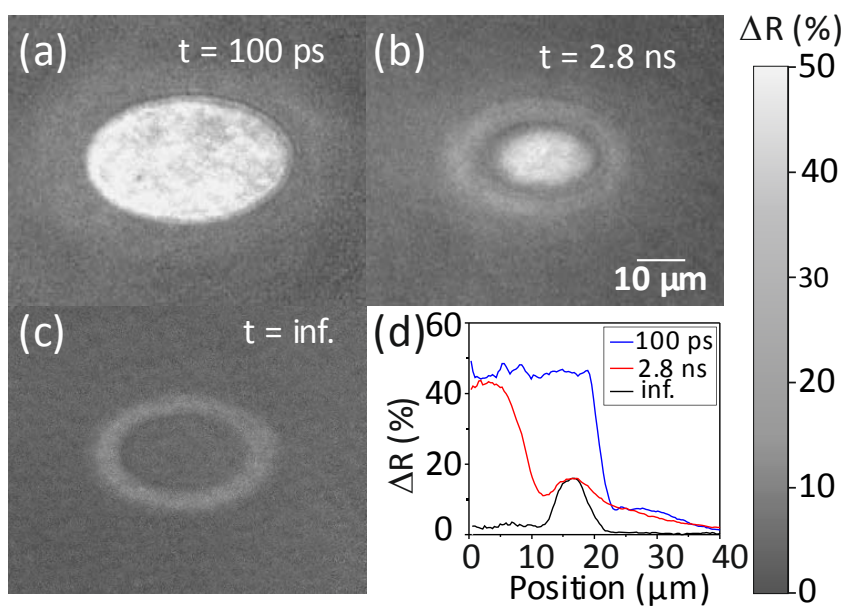

Figure 2: (a-c) Time-resolved optical micrographs of elliptical structures fabricated in $\mathrm{Si}$ by irradiation with $\mathrm{N}=3$ laser pulses at oblique incidence $\left(\theta=52^{\circ}\right)$, using a Gaussian intensity distribution with a vertical spot size of $\mathrm{d}_{\mathrm{y}}=59 \mu \mathrm{m}$ and a peak fluence $\mathrm{F}=225$ $\mathrm{mJ} / \mathrm{cm}^{2}$. The image sequence corresponds to the measurement of the material response to the third pulse. The images have been normalized with respect to the unexposed sample. Delay times are indicated in the figures and $t=$ inf. refers to a delay of several seconds. (d) Radial profiles through $(\mathrm{a}-\mathrm{c})$ quantifying the relative reflectivity change $\Delta \mathrm{R}$. The dimensions of the $\mathrm{x}$-axis refer to the horizontal axis of the images.

While Fig. 2(a) confirms the presence of melting also in the central region, the image recorded at $t=2.8 \mathrm{~ns}$ (Fig. 2(b)) shows that the liquid phase remains longer in the central region compared to the annular region, where the material has already solidified, featuring the amorphous ring. This long lifetime of the melt ${ }^{29}$ is the underlying reason for crystallization to occur in the central region. This finding is consistent with the work of several groups who reported large melt depths at high fluences, slowing down solidification and effectively enabling re-crystallization,$\underline{70}$. In contrast amorphization has been reported to occur for moderate fluences, leading to shallow melt depths and strong undercoolings. The resulting interface velocities vint are too high for a crystalline lattice to form $\left(v_{i n t}>13 \mathrm{~m} / \mathrm{s}\right)^{\underline{29}}$. This characteristic behavior of amorphization to take place at a lower local fluence (annular structure) and re-crystallization at higher fluence (central disk) is nicely observed in our results. Yet, it has to be kept in mind that the present situation corresponds to multiple pulse irradiation and is consequently more complex than the case of the single pulse irradiation studies reported in $\underline{7,29}$.

In order to investigate a possible contribution of surface ablation to the features shown, topography measurements have been performed with an optical profiler (Sensofar Pl $\mu 2300$ ), using a $50 \times$ objective lens (N.A. $=0.55)$. While this technique provides only a moderate lateral resolution $\left(R_{x y}=600 \mathrm{~nm}\right)$ the vertical resolution of the system is $R_{z}<<1 \mathrm{~nm}$. Fig. 1(f) shows cross sections through the center of the irradiations in Fig. 1(a-d). Interestingly, for $\mathrm{N}=$ 5 and 10 an annular surface depression of $\left(s d_{\max }=4 \mathrm{~nm}\right)$ can be observed, which is consistent with the annular reflectivity distribution. This implies that amorphization is accompanied by a very small surface depression, whereas the central disk remains at the same level as the surrounding surface. While this unchanged surface level in the central region unambiguously demonstrates the absence of ablation, it raises the question why the amorphous region features surface depression. This appears particularly surprising since the amorphous phase is generally considered less dense than the crystalline phase (by up to $2.2 \%$, depending in the state of structural relaxation ${ }^{1}$ ), which is why surface swelling would be expected rather than depression. Yet, it has to be said that a few works based on simulations predict the existence 
of a high density amorphous phase ${ }^{31}$. However, these few works, reporting a coordination number of 5 or higher, are limited to a-Si obtained applying extraordinarily high pressures (several tens of GPa). While such pressures are unlikely in our conditions, working below the ablation threshold, we can't rule out the possibility of a high density amorphous phase.

As an alternative - and simpler - scenario, surface depression can be caused by material evaporation taking place at the high temperatures involved, as reported by Tsibidis et $a l^{\underline{32}}$. The fact that no signs of material removal are observed in the central region for $\mathrm{N} \leq 10$, despite being exposed to an even higher local fluence might be due to the higher absorption coefficient of a-Si at the excitation wavelength ${ }^{25}$. After the first few pulses, the thin amorphous layer formed in the annular region is expected to present stronger absorption than the central region and, consequently, reaches sufficiently high temperatures for surface evaporation to take place. Yet, this increased heating for consecutive pulses in the annular region seems not to significantly slow down solidification, still leading to the formation of the amorphous phase. A detailed investigation of the complex interplay between pulse number and phase-dependent absorption is, though, beyond the scope of the present paper.

Micro-Raman measurements have been performed in order to confirm the crystalline structure of the central disk. As shown in $\frac{22}{2}$, micro-Raman spectroscopy is a suitable highsensitivity technique to study the presence of the amorphous phase in laser irradiated crystalline silicon, even if present only in form of a thin surface layer. To this end, a Renishaw InVia instrument was employed, using an excitation laser at $422 \mathrm{~nm}$ in order to obtain intense bands from both the amorphous and crystalline phase of Si. Single point spectra were obtained by using a laser power at the sample of $0.1 \mathrm{~mW}$ focused to a spot diameter of $\mathrm{d}=1 \mu \mathrm{m}$ and using an objective $100 \times$, N.A. $=0.85$. Raman maps of $25 \times 25 \mu \mathrm{m}^{2}$ were recorded by using the Stream-Line TM mode at $532 \mathrm{~nm}$ excitation, providing 600 spectra in $10 \mathrm{~min}$. for an overall laser power of $25 \mu \mathrm{W}$ extended over a line focus of $1 \times 12$ $\mu \mathrm{m}^{2}$. Fig. 3 shows the annular structure from Fig. 1(b) together with two corresponding Raman maps, which display the intensity of the Raman bands centered at $520.5 \mathrm{~cm}^{-1}$ (green map) and $473 \mathrm{~cm}^{-1}$ (red map), characteristic for crystalline and amorphous silicon, respectively 22 . The plot below (Fig. 3(d)) shows representative Raman spectra recorded outside the irradiated region, inside the annular region and within the central disk. It emerges that both the outer region and central disk feature the characteristic narrow peak of c-Si alone, while the spectrum of the annular region shows an additional Raman emission band in the 430 - $490 \mathrm{~cm}^{-1}$ region, corresponding to a-Si. The fact that the latter spectrum shows only a very weak band at $473 \mathrm{~cm}^{-1}$ and still a strong contribution from c-Si is caused by the small thickness of the amorphous surface layer (12 nm, c.f. Fig. 1(e)). Detection of the amorphous layer is especially challenging when considering the focal depth of about $2 \mu \mathrm{m}$ of the confocal Raman microscope, effectively collecting much of the Raman signal produced in the crystalline regions underneath the amorphous layer. 

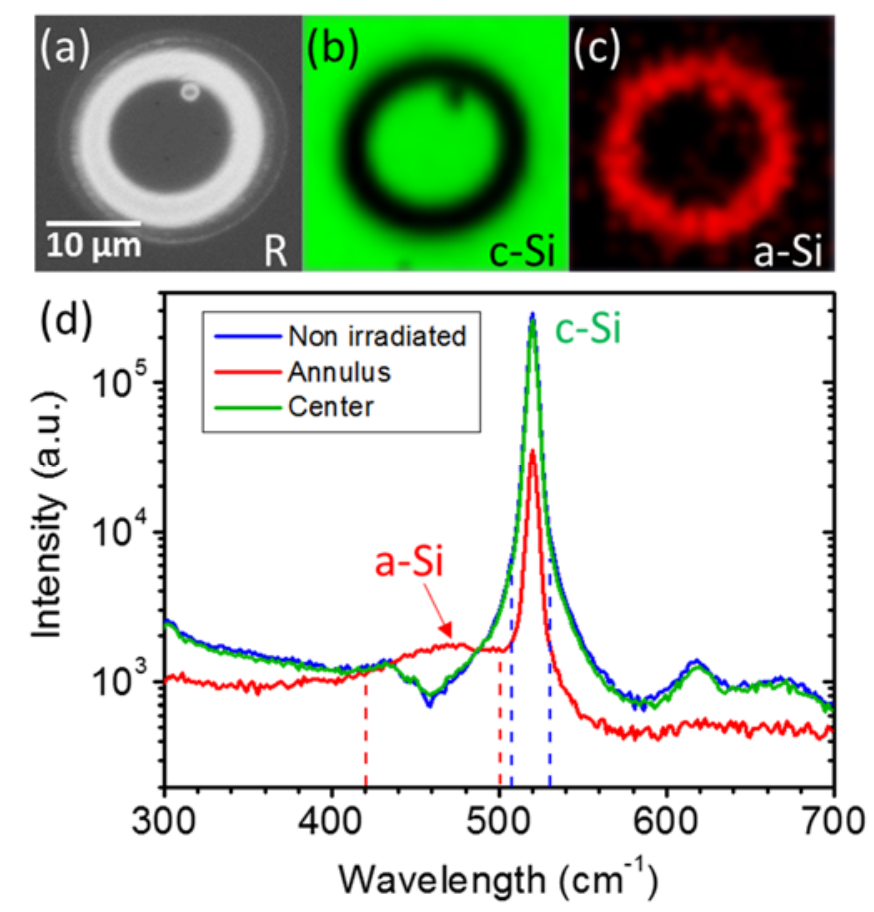

Figure 3: (a) Optical reflectivity (R) micrograph of annular structures fabricated in $\mathrm{Si}$ after irradiation with $\mathrm{N}=5$ pulses at $\mathrm{F}=250 \mathrm{~mJ} / \mathrm{cm}^{2}$. (b) Corresponding Raman map, displaying the integrated intensity of the Raman band of c-Si centered at $520.5 \mathrm{~cm}^{-1}$ and (c) for a-Si centered at $473 \mathrm{~cm}^{-1}$. (d) Raman spectra at different positions (see labels) of (a). The spectral windows over which the signal intensity has been integrated to generate the Raman maps is indicated on the horizontal axis (dashed vertical lines).

We have applied this patterning strategy to fabricate periodic arrays of microstructures with different topography patterns. Fig. 4 (a) shows the topography map of a square array of elevated discs and rhombs, produced by irradiating a $10 \times 10$ matrix at $\mathrm{N}=4$ pulses. Choosing a spacing of $25 \mu \mathrm{m}$, the surface-depressed amorphous rings overlap, forming the bottom of the pattern, as can be seen in the zoomed image of Fig. 4(b). While the elevated discs correspond to recrystallized regions similar the one shown in Fig. 1(b), the rhombs correspond to regions exposed to light intensities below the melting threshold. The equivalent crystalline state and surface height of regions exposed to fluences above the crystallization threshold and below the melting threshold allows fabricating non-annular structures. This is also illustrated in Fig. 4(c), featuring a square array of squares. The only difference in the irradiation sequence was a reduction of the spacing to $20 \mu \mathrm{m}$. Similarly, Fig. 4(d) shows a hexagonal pattern of hexagon-like structures, obtained by using again a different spacing. The topography profile corresponding to the disc structures (c.f. Fig. 4(b)) is shown in Fig. 4(e) featuring a maximum depression of $5 \mathrm{~nm}$. 

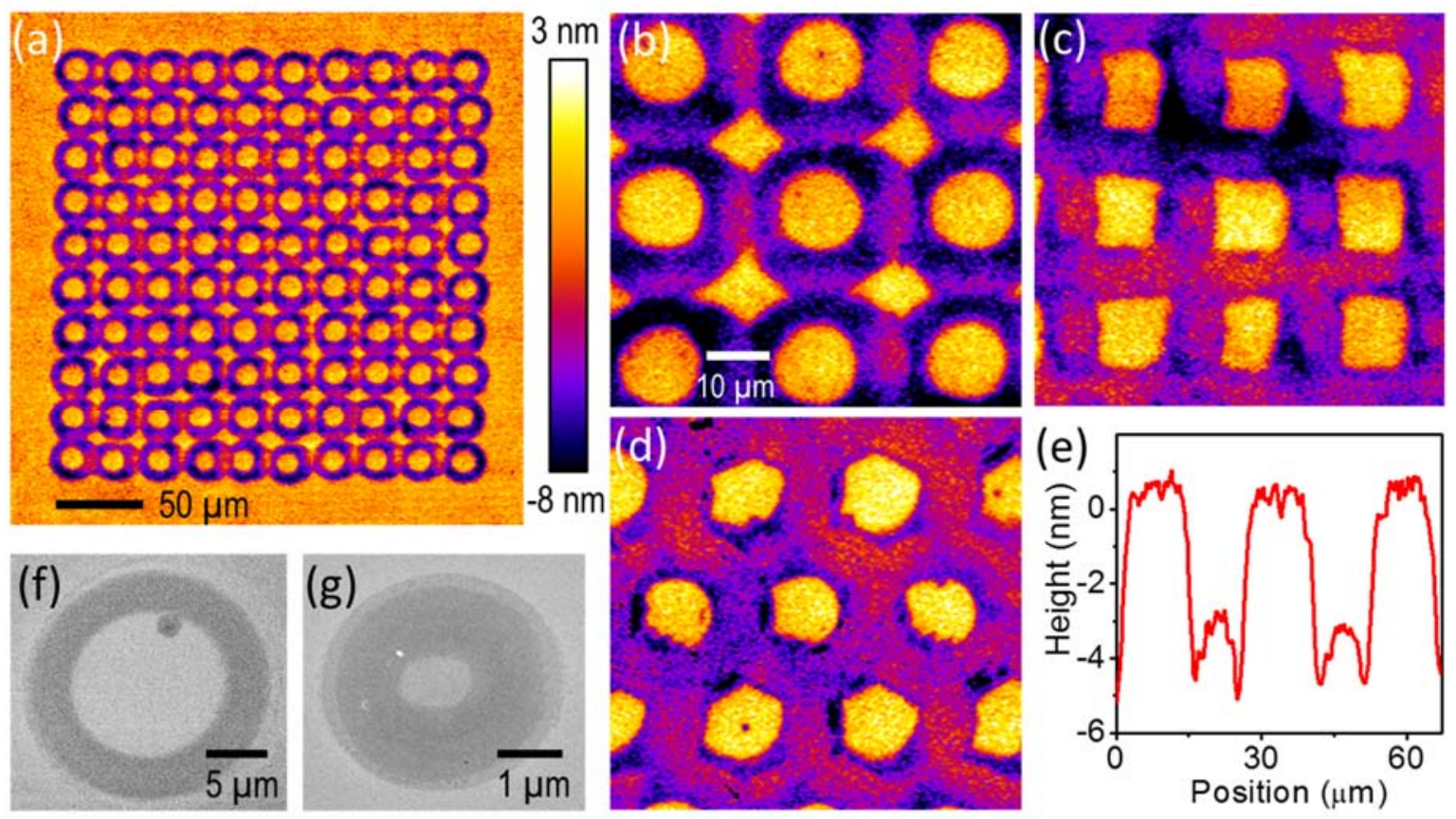

Figure 4: (a) Topography map of a 10x10 square array of irradiations with $\mathrm{N}=4$ and separation $\mathrm{x}=\mathrm{y}=25 \mu \mathrm{m}$. (b) zoomed region of (a). (c) zoomed region of an equivalent array as a but using $\mathrm{N}=3$ and $\mathrm{x}=\mathrm{y}=20 \mu \mathrm{m}$. (d) zoomed region of a hexagonal array using $\mathrm{N}=4$ $\mathrm{x}=23 \mu \mathrm{m}$ and $\mathrm{y}=19.5 \mu \mathrm{m}$. (e) horizontal profile through the center disk row of (b). (f) SEM image of the $\mathrm{N}=5$ structure shown in Fig. 1(b) (g) SEM image of a $\mathrm{N}=5$ structure produced by using an objective lens for focusing.

As a last aspect we have investigated the possibility of downscaling the ring structure. For this purpose, we have used an objective lens $(10 \times$, N.A. $=0.26)$ to focus the laser onto the surface. As can be seen in the SEM image (c.f. Fig. 4(g)), a central crystalline disk with a diameter not exceeding $1 \mu \mathrm{m}$ is produced (for comparison a SEM image of the large spot in Fig. 1(b) is displayed in Fig 4(f)). The sharp outline of the disk suggests that further downscaling may be possible and is not limited by the material response.

In conclusion, we have studied the formation of amorphous micro-structures in crystalline Si wafers using irradiation with multiple femtosecond laser pulses. These structures are formed upon melting and rapid solidification into the amorphous phase, as unambiguously demonstrated by micro-Raman spectroscopy and fs microscopy, and are characterized by a pronounced optical contrast. The thickness of the amorphous layer is found to increase as a function of laser fluence and pulse number, and can be determined precisely by means of an analysis of the reflectivity distribution. Topography studies show that the formation process does not involve surface ablation but minor evaporation. We have exploited this material response to fabricate surface-depressed annular amorphous rings with an elevated crystalline central disk that can readily be produced with few pulses at low fluence, without the need for beam shaping. The patterning strategy can be extended in two dimensions, stitching together annular structures. Exploiting the fact that the regions exposed to fluences above the crystallization threshold and below the melting threshold have the same crystalline state and surface height allows the fabrication of complex periodic patterns, featuring shapes of the unit cell that are not limited to annular structures. The simplicity of the fabrication approach combined with the fact that spatial features and optical properties can be tailored makes these 
structures to be promising candidates for applications in numerous fields, including optics, nanoelectronics, and mechatronics.

\section{Acknowledgements}

This work has been supported by the LiNaBioFluid project (H2020-FETOPEN-20142015RIA, grant 665337) of the European Commission, as well as the research grant (TEC2014-52642-C2-1-R) from the Spanish Ministry of Economy and Competitiveness. M.G.-L thanks the Spanish Ministry of Education for a FPU fellowship. The authors are grateful to C. Dorronsoro for pointing out a Matlab script for flattening the background of the data shown in Figure 4 and helping with its implementation.

\section{References}

1 E. Holmström, B. Haberl, O. H. Pakarinen, K. Nordlund, F. Djurabekova, R. Arenal, J. S. Williams, J. E. Bradby, T. C. Petersen, and A. C. Y. Liu, Journal of NonCrystalline Solids 438, 26 (2016). Khalid Laaziri, S. Kycia, S. Roorda, M. Chicoine, J. L. Robertson, J. Wang, and S. C. Moss, Physical Review B 60 (19), 13520 (1999). 
Mario Garcia-Lechuga, Daniel Puerto, Yasser Fuentes-Edfuf, Javier Solis, and Jan Siegel, ACS Photonics 3 (10), 1961 (2016). 